REGARDS

SUR L'ECONOMIE ALLEMAND

BULLETIN ECONOMIQUE DU CIRAC
Regards sur l'économie allemande

Bulletin économique du CIRAC

$88 \mid 2008$

Varia

\title{
Préserver la confiance
}

Isabelle Bourgeois

\section{OpenEdition}

Journals

Édition électronique

URL : http://journals.openedition.org/rea/2763

DOI : 10.4000/rea. 2763

ISBN : 978-2-8218-0872-0

ISSN : 1965-0787

Éditeur

CIRAC

Édition imprimée

Date de publication : 1 octobre 2008

Pagination : 3-4

ISSN : 1156-8992

Référence électronique

Isabelle Bourgeois, «Préserver la confiance », Regards sur l'économie allemande [En ligne], 88 | octobre 2008, mis en ligne le 12 janvier 2009, consulté le 15 septembre 2020. URL : http://

journals.openedition.org/rea/2763 


\section{Préserver la confiance}

L'ampleur brusquement prise début octobre par la crise de la banque Hypo Real Estate en Allemagne puis la déprime des places financières mondiales brouillent actuellement la lisibilité de l'évolution conjoncturelle outre-Rhin. Si jusqu'ici, l'économie avait assez bien résisté à la flambée des prix pétroliers, au coût des matières premières, à l'euro fort comme aux turbulences de la finance mondiale, le fait que le gouvernement fédéral ait annoncé le 5 octobre qu'il garantissait l'épargne a fait prendre conscience que la finance allemande est à son tour victime de la contagion. Entraînés par un mainstream catastrophiste, nombre d'observateurs en concluent à une crise de l'économie réelle - trop hâtivement, peut-être.

Il est certes difficile en ce moment de faire la part des choses dans le contexte de réflexions européennes crispées et placées sous le signe de l'urgence. Inquiétudes et incertitudes (récession ou pas ?) sont d'autant plus volontiers relayées par les médias que le début de l'automne est traditionnellement marqué par une pénurie d'informations factuelles et récentes sur l'évolution de l'économie. Les dernières données connues avaient été publiées par l'Office fédéral des statistiques à la fin août ; elles sont relatives au second trimestre 2008. Les instituts de conjoncture se préparent en effet à rendre leur rapport conjoint d'automne, qui sera publié le 14 octobre ; d'ici là, aucun chiffre ne filtrera. Quant au Conseil des Sages, il ne remettra son rapport annuel qu'à la mi-novembre. Cette saison centrée sur les prévisions favorise donc plus les conjectures que l'analyse sereine.

Or dans ce climat, un regard neutre est plus que jamais requis. Même si la plupart des indicateurs disponibles remontent à l'été, ils n'en permettent pas moins, si on les replace dans leur contexte structurel, de dégager une tendance. Comme toutes les autres économies de la zone euro et de l'UE, l'Allemagne affronte maintenant un net ralentissement des activités. Un tassement à partir du milieu de l'année était attendu depuis plusieurs mois déjà ; ces prévisions incluaient aussi l'incertitude quant à l'ampleur qu'il prendrait (voir REA 87/08). La Commission européenne et l'OCDE ont récemment revu à la baisse leurs prévisions de croissance pour la zone euro, qui devrait être de $+1,3 \%$ (au lieu de $+1,7 \%$ ). Quant aux prévisions 2009, elles sont quasi impossibles à établir actuellement, comme le révèle la fourchette avancée en octobre par la BCE : elle va de $+0,6 \%$ à $+1,8 \%$. En 2008 en tout cas, l'économie allemande, qui 'pèse' un bon quart dans celle de la zone euro, devrait bien tirer son épingle du jeu, avec une croissance que la Commission européenne estime à $+1,8 \%$ ('OCDE pour sa part, à $+1,5 \%$ seulement). Les prévisions des instituts économiques allemands, distillées ça et là, se situent dans la même fourchette.

Selon Destatis, la croissance du $2^{\mathrm{e}}$ trimestre 2008 a été négative $(-0,5 \%)$ par rapport au précédent. Elle n'en était pas moins en hausse $(+3,1 \%)$ par rapport au $1^{\mathrm{er}}$ trimestre 2007. C'est sur ce constat que milieux économiques et pouvoirs politiques fondaient jusqu'ici leur confiance dans la robustesse foncière des activités, d'autant que le recul trimestriel n'était pas imputable à la balance commerciale (excédentaire), mais aux investissements dans la construction dont la chute $(-3,5 \%)$ ne traduisait en réalité qu'un retour à la normale après un hiver particulièrement doux. Au total, alors que les dépenses de consommation privées avaient baissé (-0,7\% en trois mois) du fait de la hausse des prix, c'est toujours l'excédent commercial qui tirait l'activité (il est entré pour 0,4 point dans la création de richesse).

Mais l'économie réelle de l'Allemagne ressent dorénavant - avec un léger temps de retard sur les autres Etats de l'UE - les effets de l'euro fort et de la flambée des prix pétroliers comme des matières premières, celle-ci ayant contribué entre-temps à tasser la demande mondiale et à maintenir, au sein de la zone euro, un taux d'inflation élevé. La variation des carnets de commande de l'industrie en est un premier signe avant-coureur: selon le ministère fédéral de l'Economie, qui s'appuie sur des données de Destatis, en juillet/août 2008, les commandes ont diminué de $0,8 \%$ (données CVS) par rapport à la même période de 2007. La baisse est imputable un peu moins au recul de la demande intérieure $(-0,7 \%)$ qu'à celui de la demande étrangère $(-0,9 \%)$. «Les perspectives pour la production industrielle seront moins favorables au cours des prochains mois », conclut prudemment le ministère (communiqué, 07-10-08). La Fédération de la construction mécanique (VDMA), pour sa part, annonce qu'en août les entrées de commandes étrangères ont baissé de $19 \%$ par rapport à août 2007, alors que les commandes domestiques ont augmenté de $6 \%$. Mais cette manifestation du ralentissement de l'activité mondiale ne semble pas inquiéter outre mesure la branche qui s'attend à une croissance de $5 \%$ de la production d'ici la fin de l'année, prolongeant une hausse qui se maintient depuis cinq ans; ses stocks de commandes sont élevés. Quant à l'industrie automobile, mise à mal au fil des mois par la baisse du pouvoir d'achat des Allemands, mais aussi des Américains, et leur attentisme face au prix des carburants, elle ralentit maintenant sa production pour diminuer le volume
Pénurie de données factuelles au début de l'automne

Baisse des prévisions de croissance pour 2008

Recul de la croissance au $2^{\mathrm{e}}$ trimestre ; et au $3^{\mathrm{e}}$ ?

La crise financière amplifie maintenant les effets du tassement antérieur de l'économie réelle mondiale 
Resserrement du crédit ou non ? Les experts sont divisés

Pour diverses raisons structurelles l'emploi se maintient de ses immobilisations. Cela étant, cette tendance n'affecte que les voitures particulières (les immatriculations allemandes ont baissé de $2 \%$ en septembre par rapport au même mois de 2007). Car le segment des utilitaires, à l'inverse, a vu les immatriculations grimper de $12 \%$, les exportations augmentant de $10 \%$.

Bien que l'Allemagne soit moins tributaire du marché américain qu'avant, et que certains experts voient même clairement la Chine surtout prendre le relais, le régime des exportations, le principal moteur de l'économie allemande, commence à ralentir. Difficile cependant de faire la part des choses entre ce qui est imputable à l'évolution du contexte économique (coûts de production, parité $€ / \$$ ) et aux effets de la crise financière ; ceux-ci ne faisant vraisemblablement qu'amplifier les difficultés liées à une longue baisse de la demande dans les pays industrialisés. Les grandes fédérations allemandes sont elles-mêmes divisées dans leur analyse : alors que celle de l'industrie (BDI) pense que les entreprises vont voir se resserrer les conditions de crédit, celle des $\mathrm{CCl}$ (DIHK) estime au contraire que la crise financière va clairement bénéficier aux PME, puisqu'elle amène les banques allemandes à se recentrer sur les investissements à long terme, c'est-à-dire le financement des PME.

Quant au marché de l'emploi, qui réagit toujours avec quelques mois de retard à l'évolution macro-économique, il reste bien orienté. De juillet 2007 à juillet 2008, le nombre d'actifs occupés a augmenté de 560000 personnes, principalement du fait de la hausse des emplois soumis à cotisations sociales (+ 596000 entre juin 2007 et 2008), explique le ministère fédéral de l'Economie. Cette embellie se maintient: en septembre, le nombre de chômeurs n'atteignait plus que 3,081 millions, le taux de chômage tombait à $7,4 \%$ - le niveau le plus bas depuis 16 ans. Cette tendance s'explique, selon Destatis, par l'extension de l'emploi atypique au cours de la décennie : alors qu'en 1997, la part des actifs occupant un emploi régulier, classique, était encore de $82,5 \%$, elle est tombée à $74,5 \%$ depuis. Cette évolution structurelle ne peut toutefois s'assimiler à une "précarisation de l'emploi » comme l'affirment volontiers les syndicats, puisque, la plupart du temps, les nouvelles formes d'emploi " sont délibérément choisies afin de concilier objectifs professionnels et privés », comme l'explique Destatis (Welt online, 09-09-08). Les experts sont optimistes quant à la bonne tenue du marché du travail pour une autre raison encore: le manque de maind'œuvre qualifiée a amené les entreprises à changer leur GRH : elles évitent de licencier, sachant ce qu'il leur en coûte de recruter de nouveaux salariés, surtout au moment où les mutations démographiques commencent à réduire globalement l'offre de main-d'œuvre.

LES FONDAMENTAUX ESSENTIELS DE L'ECONOMIE REELLE RESTENT BONS. En interne, le tassement de la demande mondiale affecte l'activité au moment même où, sur fond d'une dynamique de croissance ininterrompue depuis 2006 , les réformes structurelles commencent à porter leurs fruits, se traduisant par une bonne tenue du marché de l'emploi. A l'extérieur, le leadership technologique conquis par de nombreux acteurs de l'industrie allemande sur le marché mondial leur permet de répercuter en grande partie la hausse des coûts de production sur leurs clients étrangers. "C'est la raison pour laquelle les entreprises allemandes résisteront aux mouvements tectoniques déclenchés par la crise des marchés financiers et en sortiront avec une compétitivité internationale accrue », affirme Thomas Straubhaar, directeur de l'institut HWWI de Hambourg (Standpunkt, 30-09-08). S'ajoute à cela que le retour à un niveau plus raisonnable à la fois des prix pétroliers, du coût des matières premières et de la parité $€ / \$$, si cette tendance se maintient, créera un environnement nettement plus favorable à l'activité pour la zone euro notamment. Reste la question d'une consommation intérieure largement bridée par un pouvoir d'achat grevé par un taux d'inflation qui, après un pic de 3,3\% en juin et juillet, est retombé depuis à $3 \%$. Effet du début de la baisse des prix de l'énergie, du carburant et des produits alimentaires, la décrue devrait s'accélérer maintenant. A une condition toutefois: que les syndicats allemands, et plus particulièrement IG Metall, renouent dans leurs négociations en cours avec leur modération salariale passée. Or le risque d'un 'second tour' en Allemagne était l'une des raisons pour lesquelles la BCE se refusait à baisser son taux directeur. La nécessité absolue de préserver la confiance au sein de la zone euro l'a emporté le 8 octobre sur l'impératif de stabilité des prix et l'a amenée à l'abaisser de 0,5 point.

C'est justement parce que les fondamentaux de l'économie réelle restent bons qu'il convient de ne pas les fragiliser en cette période d'incertitudes financières. "L'Allemagne est bien armée pour la compétition globale. Je suis convaincue que cela nous aidera à maîtriser l'impact de la crise financière, même si cela ne sera pas facile », déclarait la chancelière Angela Merkel le 7 octobre devant le Bundestag. II ne faut pas céder aux voix de Cassandre, mais bien au contraire poursuivre sur la voie des réformes structurelles pour que l'économie allemande puisse continuer à donner sa pleine mesure. C'est là la seule réponse politique possible pour « recréer et conforter la confiance » de tous les acteurs.

Isabelle Bourgeois (08-10-2008) 